\title{
Protein disulfide isomerase inhibition blocks thrombin generation in humans by interfering with platelet factor $\mathrm{V}$ activation
}

\author{
Jack D. Stopa, ${ }^{1}$ Donna Neuberg, ${ }^{2}$ Maneka Puligandla, ${ }^{2}$ Bruce Furie, ${ }^{1}$ Robert Flaumenhaft, ${ }^{1}$ \\ and Jeffrey I. Zwicker \\ 'Division of Hemostasis and Thrombosis, Beth Israel Deaconess Medical Center and Harvard Medical School, \\ ${ }^{2}$ Department of Biostatistics and Computational Biology, Dana Farber Cancer Institute, Boston, Massachusetts, USA
}

BACKGROUND: Protein disulfide isomerase (PDI) is required for thrombus formation. We previously demonstrated that glycosylated quercetin flavonoids such as isoquercetin inhibit PDI activity and thrombus formation in animal models, but whether extracellular PDI represents a viable anticoagulant target in humans and how its inhibition affects blood coagulation remain unknown.

METHODS: We evaluated effects of oral administration of isoquercetin on platelet-dependent thrombin generation in healthy subjects and patients with persistently elevated anti-phospholipid antibodies.

RESULTS: Following oral administration of $1,000 \mathrm{mg}$ isoquercetin to healthy adults, the measured peak plasma quercetin concentration $(9.2 \mu \mathrm{M})$ exceeded its $\mathrm{IC}_{50}$ for inhibition of PDI by isoquercetin in vitro $(2.5 \pm 0.4 \mu \mathrm{M})$. Platelet-dependent thrombin generation decreased by $51 \%$ in the healthy volunteers compared with baseline $(P=0.0004)$ and by $64 \%$ in the anti-phospholipid antibody cohort $(P=0.015)$ following isoquercetin ingestion. To understand how PDI affects thrombin generation, we evaluated substrates of $\mathrm{PDI}$ identified using an unbiased mechanistic-based substrate trapping approach. These studies identified platelet factor $\mathrm{V}$ as a PDI substrate. Isoquercetin blocked both platelet factor $\mathrm{Va}$ and thrombin generation with an $\mathrm{IC}_{50}$ of $\sim 5 \mu \mathrm{M}$. Inhibition of PDI by isoquercetin ingestion resulted in a $53 \%$ decrease in the generation of platelet factor $\operatorname{Va}(P=0.001)$. Isoquercetin-mediated inhibition was reversed with addition of exogenous factor Va.

CONCLUSION: These studies show that oral administration of isoquercetin inhibits PDI activity in plasma and diminishes platelet-dependent thrombin generation predominantly by blocking the generation of platelet factor Va. These pharmacodynamic and mechanistic observations represent an important step in the development of a novel class of antithrombotic agents targeting PDI.

TRIAL REGISTRATION: Clinicaltrials.gov (NCT01722669)

Role of funding source: NHLBI supported all aspects of study. Quercegen Pharma provided isoquercetin and funding support for pharmacokinetic analyses.

Authorship note: R. Flaumenhaft and J.I. Zwicker contributed equally to this work.

Conflict of interest: J.I. Zwicker received research support from Quercegen Pharma.

Submitted: July 11, 2016 Accepted: November 29, 2016 Published: January 12, 2017

Reference information: JCI Insight. 2017;2(1):e89373. doi:10.1172/jci.insight.89373.

FUNDING: National Heart, Lung, and Blood Institute (U54 HL112302) and Quercegen Pharma

\section{Introduction}

Protein disulfide isomerase (PDI) is a ubiquitously expressed thiol isomerase found in high concentrations in the endoplasmic reticulum (ER), where it serves an essential role in protein folding through disulfide bond shuffling. However, PDI can escape ER retention mechanisms in several cell types and localize to secretory granules and membrane surfaces. Within the vasculature, platelets and endothelial cells secrete PDI in the context of vascular injury or inflammation. Several animal models have demonstrated that extracellular PDI has a critical role in thrombus formation $(1,2)$. Inhibition of PDI by either monoclonal antibodies or small-molecule inhibitors prevents both platelet accumulation and fibrin formation at sites of vessel injury (2-4). Thrombus formation is also impaired in mice deficient in platelet PDI (5).

Flavonoid quercetins are among the small-molecule inhibitors of PDI that block thrombus formation. These flavonoids were first identified as PDI inhibitors in a high-throughput screen of an annotated library of known bioactive compounds that identified quercetin-3-rutinoside (also called rutin) as an inhibitor 
of PDI activity (6). Quercetin-3-rutinoside inhibited platelet aggregation and injury-induced fibrin formation on endothelium in cell-based assays. The administration of quercetin-3-rutinoside similarly inhibited thrombus formation following vascular injury in mouse models of thrombosis (6). Quercetin-3-rutinoside blocks PDI activity by binding to the substrate-binding pocket on PDI and inducing a conformational change in the enzyme, which results in a more compact molecular envelope and reduces substrate binding (7). Structure-activity relationship assays showed that all quercetins tested that possessed a glycoside at the third position on the $\mathrm{C}$ ring inhibited PDI, including isoquercetin (6) (also known as quercetin-3-glucoside), which has improved bioavailability in humans compared with quercetin-3-rutinoside (8-11). Interestingly, quercetins are flavonoids present in a wide variety of natural foods (e.g., fruits and vegetables), and a number of epidemiologic studies point to potential cardiovascular benefits of diets rich in quercetins. For instance, in the Zutphen Elderly study, high dietary consumption of quercetins was associated with a $70 \%$ decrease in mortality secondary to myocardial infarction (12). Consumption of quercetin has similarly been linked to a decrease in fatal and nonfatal cerebrovascular accidents (13).

Regulation of vascular cell surface receptors and coagulation proteins by modification of disulfide bonds represents a previously unrecognized layer of control of thrombus formation, akin to posttranslational modification by proteases. Unlike the role of proteases in blood coagulation, which has been studied in depth, little is known about the substrates and posttranslational modifications performed by PDI and other thiol isomerases. Several platelet and endothelial integrins such as $\mathrm{Ib} \alpha(14), \alpha \operatorname{Ilb} \beta 3$ (15), and $\alpha \mathrm{Vb} \beta 3$ (16) are identified as putative substrates for PDI, but how PDI modifies these substrates is not well understood. Still less is known regarding which coagulation factors are directly targeted by extracellular PDI. A disulfide bond within factor XI can be reduced by PDI in vitro (17) and the Cys186-Cys209 disulfide bond in tissue factor may be susceptible to PDI-mediated thiol exchange, although this hypothesis remains controversial (18-21).

Following the discovery of a role for PDI in thrombus formation and the identification of quercetin3-rutinoside as a small-molecule inhibitor of PDI activity with an established safety profile in humans, we initiated pharmacokinetic and pharmacodynamic clinical studies with quercetin analogs (quercetin aglycone and isoquercetin). Based on prior observations that PDI inhibition diminishes platelet-dependent thrombin generation in vitro, we mechanistically evaluated whether the oral administration of isoquercetin diminishes platelet-dependent generation of thrombin in humans in a PDI-dependent manner. Utilizing an unbiased strategy to identify novel substrates using PDI trapping variants (22), we now identify platelet factor $\mathrm{V}$ as a principal coagulation substrate of extracellular PDI. These studies provide therapeutic and mechanistic insights into a potentially novel class of antithrombotic drugs targeting PDI.

\section{Results}

Inhibition of PDI in human subjects using isoquercetin. Initial studies were designed to determine whether the oral administration of a quercetin flavonoid achieved plasma drug levels known to inhibit PDI activity in vitro (Figure 1). Accordingly, we evaluated whether the presence or absence of a glycoside moiety at the 3-C position of quercetin influenced absorption. We administered $500 \mathrm{mg}$ quercetin aglycone or $500 \mathrm{mg}$ isoquercetin orally to healthy adults. Venipuncture was performed prior to quercetin ingestion and then serially over 24 hours and total quercetin levels in plasma were measured. Quercetin aglycone (500 mg) ingestion yielded a median peak concentration $\left(\mathrm{C}_{\max }\right.$ ) of $0.8 \mu \mathrm{M}$ and an AUC of $3.8 \mu \mathrm{M} \mathrm{hr} / 1$ (Figure 2A), whereas with 500 mg isoquercetin the $\mathrm{C}_{\max }$ was $4.22 \mu \mathrm{M}$ with an AUC of $18.3 \mu \mathrm{M} \mathrm{hr} / 1$ (Figure 2B). Both quercetin analogs were formulated with ascorbic acid in order to prevent oxidation during storage. The addition of ascorbic acid did not influence the pharmacokinetic profile of either quercetin aglycone (AUC of $5.1 \mu \mathrm{M}$ hr/1, Wilcoxon signed-rank test $P=0.31$ ) or isoquercetin (AUC of $13.6 \mu \mathrm{M} \mathrm{hr} / 1$, Wilcoxon signed-rank test $P=1.00$ ) (Figure 2). The highest isoquercetin dose we tested was $1,000 \mathrm{mg}(n=10)$, which yielded a significantly higher peak plasma quercetin concentration $(9.2 \mu \mathrm{M})$ and AUC (39.4 $\mu \mathrm{M} \mathrm{hr} / 1)$ compared with the isoquercetin 500-mg cohort (Mann-Whitney rank-sum test $P=0.03$ for both variables) (Figure 2).

We developed a plasma-based assay to measure PDI inhibition following ingestion of flavonoids utilizing a fluorescence-based probe, di-eosin-GSSG, which consists of 2 eosin moieties coupled to glutathione disulfide (GSSG). Cleavage of di-eosin-GSSG by recombinant PDI results in liberation of the eosin moieties with a concomitant increase in fluorescence. As shown in Figure $2 \mathrm{C}$, the ex vivo addition of quercetin-3-rutinoside, isoquercetin, or an abundant metabolite, quercetin-3-glucuronide, to plasma resulted in a dose-dependent inhibition of PDI activity in vitro. The $\mathrm{IC}_{50}$ for PDI inhibition was $2.5 \pm 0.4 \mu \mathrm{M}$ for isoquercetin, $8.5 \pm 2.1 \mu \mathrm{M}$ for quercetin-3-glucuronide, and $8.9 \pm 2.3 \mu \mathrm{M}$ for quercetin-3-rutinoside. 


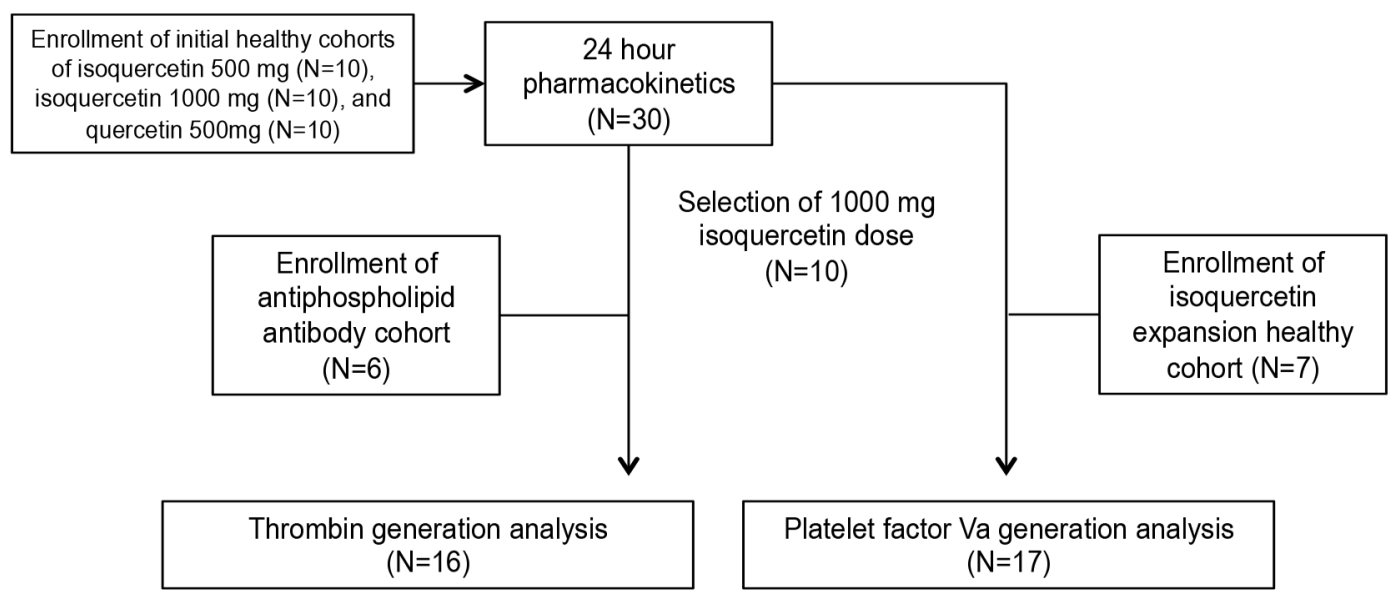

Figure 1. Flow diagram of cohort enrollment and sample analysis.

Using this PDI activity assay, we evaluated the pharmacodynamic inhibition of PDI activity over time by subjects who had ingested 1,000 mg isoquercetin. Inhibition of PDI activity mirrored the total quercetin concentrations in plasma (Figure 2D). We observed a significant 38\% (95\% CI $12.4 \%$ to $63 \%$ ) reduction in PDI activity in subject plasmas by 2 hours following the administration of $1,000 \mathrm{mg}$ isoquercetin compared with plasma obtained prior to isoquercetin ingestion $(P=0.03)$. In contrast, the ability of subject plasma to reduce PDI activity was not significantly changed 2 hours following ingestion of either $500 \mathrm{mg}$ quercetin aglycone or $500 \mathrm{mg}$ isoquercetin compared with plasmas obtained prior to flavonoid quercetin administration ( $P=0.98$ and $P=0.68$, respectively). Accordingly, peak plasma quercetin concentration correlated with peak PDI inhibition (Spearman correlation coefficient $0.55, P=0.002$ ). At peak quercetin concentrations above $4 \mu \mathrm{M}$, the median inhibition of PDI was $0.69 \mathrm{U} / \mathrm{ml}$ compared with $0.29 \mathrm{U} / \mathrm{ml}$ for lower peak quercetin concentrations $(P=0.002$, Figure $2 \mathrm{E})$.

PDI inhibition using isoquercetin blocks thrombin generation. The platelet-dependent thrombin generation assay was designed to mimic thrombin propagation through platelet activation with low-dose thrombin (23). Increased platelet-dependent thrombin generation has been observed in hypercoagulable states $(24,25)$, and hemorrhagic conditions are associated with decreased activity $(26,27)$. In healthy individuals following 1,000 mg isoquercetin administration $(n=10)$, we observed a significant $51 \%$ reduction in thrombin generation at 4 hours $(0.30 \mathrm{U} / \mathrm{ml}, 95 \%$ CI $0.09-0.50 \mathrm{U} / \mathrm{ml})$ compared with baseline $(0.59 \mathrm{U} / \mathrm{ml}, 95 \%$ CI $0.39-0.80 \mathrm{U} / \mathrm{ml}$, $P=0.0004$ ) (Figure 3B). Although no significant reduction in thrombin generation was observed in subject plasmas following a $500 \mathrm{mg}$ dose of isoquercetin compared with baseline $(P=0.22)$, we observed a significant correlation between plasma quercetin levels and thrombin generation (Spearman correlation coefficient $-0.34, P=0.02$ ). Plasma quercetin levels above $4 \mu \mathrm{M}$ were associated with significantly lower platelet-dependent thrombin generation compared with plasma levels between 0 and $2 \mu \mathrm{M}$ (Mann-Whitney $P=0.012$ ).

To assess whether inhibition of endogenous PDI was responsible for the decrease in thrombin generation, we determined whether or not the effect of isoquercetin could be reversed by the inclusion of excess recombinant $\mathrm{PDI}$ in the reaction mixture. Healthy donor plasma was incubated with $10 \mu \mathrm{M}$ isoquercetin ex vivo followed by exposure to $50 \mu \mathrm{M}$ recombinant PDI. Inhibition of platelet-dependent thrombin generation by isoquercetin was reversed and thrombin generation normalized following incubation with excess PDI (Figure 3A). Similarly, the addition of $50 \mu \mathrm{M}$ recombinant PDI to plasma from study subjects did not increase baseline thrombin generation $(0.57 \mathrm{U} / \mathrm{ml}, 95 \% \mathrm{CI} 0.43-0.72 \mathrm{U} / \mathrm{ml})$ but restored thrombin generation at 4 hours following $1,000 \mathrm{mg}$ isoquercetin ingestion $(0.50 \mathrm{U} / \mathrm{ml}, 95 \% \mathrm{CI} 0.34-0.66 \mathrm{U} / \mathrm{ml}, P=0.001)$ to within $87 \%$ of baseline activity (Figure $3 \mathrm{~B}$ ). These results show that a molar excess of recombinant PDI is able to restore platelet-dependent thrombin generation following inhibition by isoquercetin, implicating PDI as the principal target of isoquercetin in this assay.

Prothrombin time (PT) was unaffected by the administration of isoquercetin, with a mean PT of 12.8 seconds (95\% CI 12.4-13.2 seconds) at baseline compared with 12.8 seconds (95\% CI 12.4-13.3 seconds) after 4 hours $(P=0.82)$. Similarly, thrombin times (TTs) were similar at baseline (16.8 seconds, $95 \%$ CI $15.7-17.9$ seconds) and at 4 hours (16.3 seconds, 95\% CI 14.7-17.9 seconds) following isoquercetin ingestion $(P=0.46)$. 
A

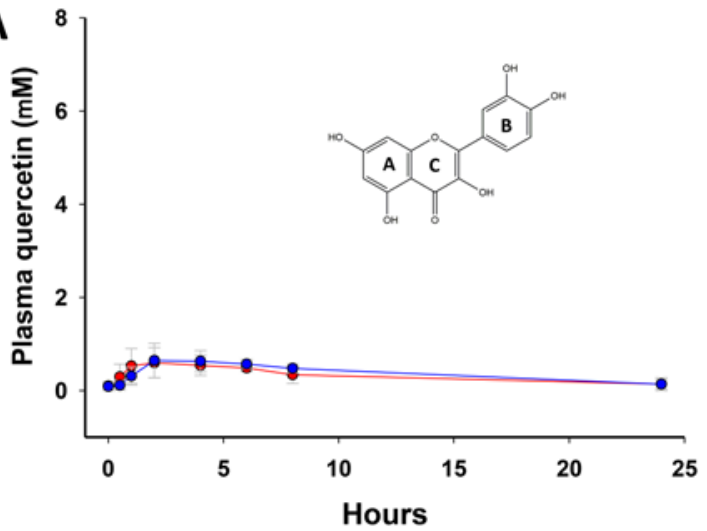

C

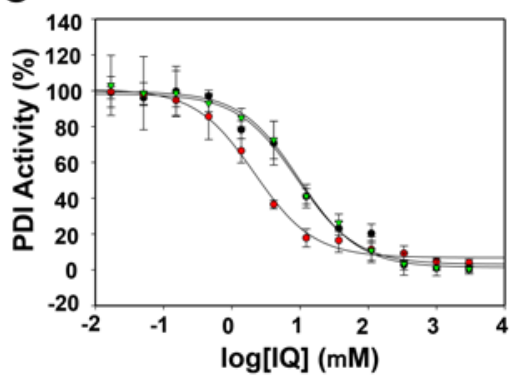

D

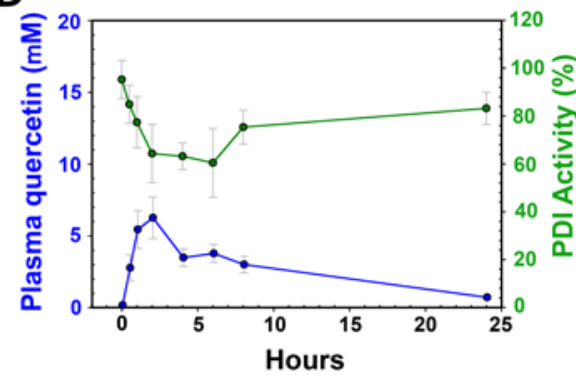

B

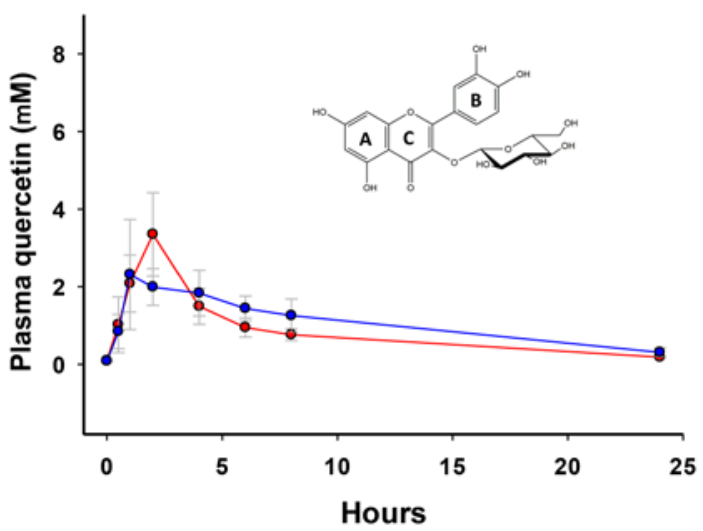

$\mathbf{E}$

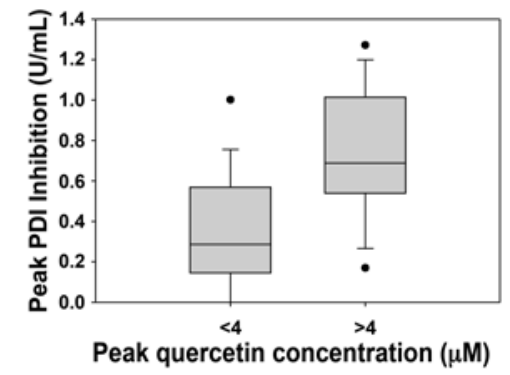

Figure 2. Isoquercetin inhibits protein disulfide isomerase activity in plasma. Levels of total plasma quercetin were determined at serial time points over 24 hours following oral administration to healthy adults of (A) $500 \mathrm{mg}$ quercetin aglycone or (B) $500 \mathrm{mg}$ isoquercetin. Quercetin levels were measured by HPLC after enzymatic hydrolysis. Quercetin analogs were formulated with ascorbic acid/niacin $(n=5$, red) and without ascorbic acid/niacin ( $n=5$, blue). Error bars represent SEM. (C) Measurement of protein disulfide isomerase (PDI) inhibition using a plasma-based assay. The indicated concentrations of isoquercetin (red), quercetin-3-glucuronide (black), or quercetin-3-rutinoside (green) were incubated with recombinant PDI in human plasma and PDI activity measured using a di-eosin-CSSG-based assay. IQ, isoquercetin. Mean values of triplicates ( \pm SEM) represent percentage of PDI activity. (D) Comparison of PDI inhibition and quercetin levels following administration of isoquercetin to healthy adults. Plasma was obtained from patients at the indicated times following ingestion of $1,000 \mathrm{mg}$ isoquercetin. Inhibition of recombinant PDI by plasma (green, scale per right $y$ axis) is presented as percentage PDI activity compared with plasma obtained prior to ingestion (time 0 ). Circles represent mean values of 10 patients with SEM shown as error bars. Quercetin concentration in plasma (blue, scale per left $y$ axis) was determined using HPLC. (E) Box-and-whisker plot of peak PDI inhibition at peak quercetin concentrations above or below $4 \mu \mathrm{M}$. Top and bottom of box represent the interquartile range along with median values, whiskers represent 95 th percentile, and outliers are shown. Mann-Whitney $P=0.002$.

Thrombin generation in patients with elevated anti-phospholipid antibodies. In order to evaluate the potential anticoagulant activity of isoquercetin in a patient population at increased risk for thrombosis, we evaluated thrombin generation in patients with persistently elevated anti-phospholipid antibodies who were not taking anticoagulants or antiplatelet agents $(n=6)$. In this cohort were 5 individuals with lupus anticoagulant and 1 patient with high-titer anti-cardiolipin IgM antibodies (above 100 IgG phospholipid [GPL]). As shown in Figure 4, baseline thrombin generation was significantly higher compared with healthy controls $(P=0.01)$. All 6 patients with anti-phospholipid antibodies had lower thrombin generation following 1,000 mg isoquercetin ingestion with a mean decrease of $63.6 \%$ in thrombin generation at 4 hours compared with baseline (4.01 U/ml vs. $1.46 \mathrm{U} / \mathrm{ml}, P=0.015)$.

PDI inhibition decreases generation of platelet factor $\mathrm{Va}$. We have previously developed an unbiased strategy to identify novel substrates of PDI in washed platelets using PDI variants capable of trapping substrates (22). This approach uses a FLAG-tagged PDI variant modified by a substitution of arginine or proline for histidine (CGHC $\rightarrow$ CGRC; CGHC $\rightarrow$ CGPC) in the catalytic motif of both the $a$ and $a^{\prime}$ domains. Although these variant PDIs remain capable of forming mixed disulfide bonds with putative substrate proteins, their ability to resolve the mixed disulfide bond is impaired and the duration of the PDI-substrate intermediate prolonged. This intermediate can be captured by alkylation of free thiols by $N$-ethylmaleimide (NEM) to prevent resolution of the mixed disulfide bond. The PDI-substrate complex is isolated using immobilized anti-FLAG antibody and subsequently identified by mass spectrometry. Using this strategy, we have now identified platelet factor V as a substrate of PDI (Figure 5). Mechanism-based kinetic trapping 
A

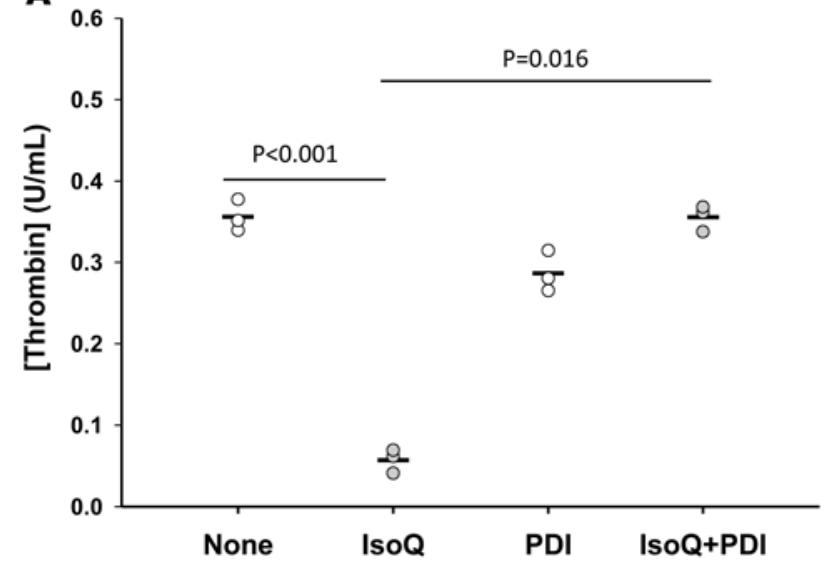

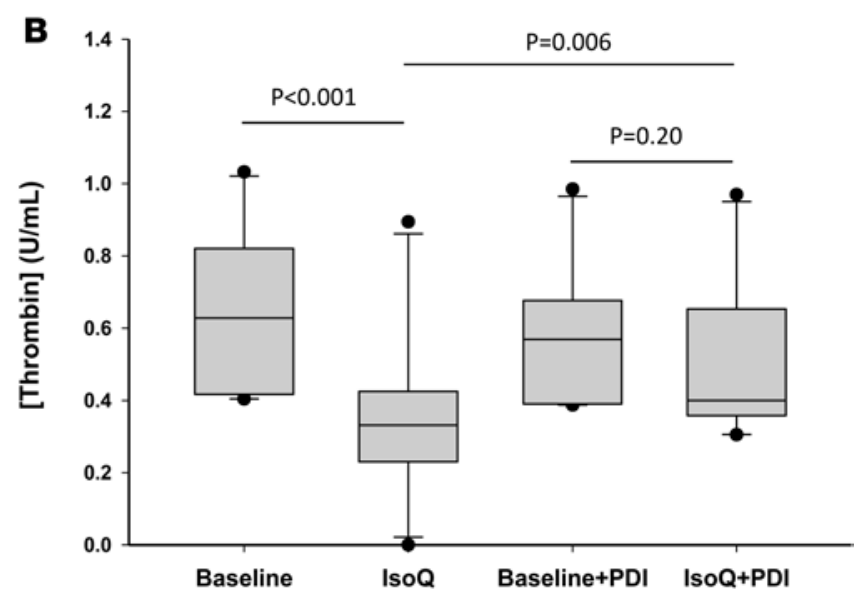

Figure 3. Thrombin generation is decreased in a protein disulfide isomerase-dependent manner following isoquercetin administration. (A) Plateletdependent thrombin generation was evaluated in platelet-rich plasma incubated with no addition (None), $10 \mu \mathrm{M}$ isoquercetin (IsoQ), $50 \mu \mathrm{M}$ protein disulfide isomerase $(\mathrm{PDI})$, or both (IsoQ+PDI). The reaction was initiated using $0.1 \mathrm{U} / \mathrm{ml}$ thrombin and platelet-dependent thrombin generation detected using a fluorescent thrombin-specific substrate. Triplicate results and mean values are represented as peak thrombin generated (U/ml) following initiation of reaction. $P$ values were determined by 1-way ANOVA with Sheffe's correction for multiplicity of comparison. (B) Ten healthy participants received an oral dose of $1,000 \mathrm{mg}$ isoquercetin and blood samples were drawn just before (baseline, PDI) and 4 hours after (IsoQ, IsoQ+PDI) ingestion. Samples were subsequently incubated in the absence (baseline, IsoQ) or presence (PDI, IsoQ+PDI) of PDI and assayed for platelet-dependent thrombin generation using a fluorescent thrombin-specific substrate. Top and bottom of box represent the interquartile range along with median values, whiskers represent 95 th percentiles, and outliers are shown $(n=10)$. $P$ values were determined using a mixed-model ANOVA with Sheffe's correction for multiplicity of testing.

was performed using both the PDI-CGPC and PDI-CGRC variants. To confirm that the interaction of PDI with platelet-derived factor $\mathrm{V}$ involves a disulfide modification and not noncovalent binding, we performed substrate trapping experiments using a FLAG-tagged PDI variant in which the catalytic cysteines in the $a$ and $a^{\prime}$ domains were mutated to alanine (CGHC $\rightarrow$ AGHA). Washed platelets were stimulated with thrombin in the presence of FLAG-tagged PDI variants. The CGRC-PDI variant coprecipitated with platelet factor $\mathrm{V}$, while there was no platelet factor $\mathrm{V}$ detected with the AGHA-PDI variant, thus confirming the direct binding of PDI with platelet-derived factor $\mathrm{V}$ by disulfide bond exchange (Figure 5A).

A special property of these variants compared with previously trapping strategies $(28,29)$ is that they are able to trap substrates regardless of whether they are reduced or oxidized by PDI. These mutants were prereduced or preoxidized to bias trapping of reduced substrates or oxidized substrates, respectively (22). A comparison of the ability of preoxidized versus prereduced forms of the mutants to trap a particular substrate provides an indication of whether PDI oxidizes or reduces the substrate. The amount of substrate trapped by the CGRC-PDI and CGPC-PDI variants are quantified by densitometry. The ratio of factor $\mathrm{V}$ trapped by the prereduced form of the variant compared with the preoxidized form of the variant (reduced/oxidized) was 2.88 for CGRC-PDI and 1.29 for CGPC. Thus, the trapping mutants were more effective following reduction than following oxidation, indicating that PDI reduces platelet factor V (Figure 5A). Evaluation of trapping experiments using agarose gels to identify high-molecular-weight complexes demonstrated that factor $\mathrm{V}$ from platelets is present in a high-molecular-weight complex with multimerin (Figure 5B).

To evaluate the effect of PDI inhibition on the activation of factor V, washed platelets from healthy donors were stimulated with $0.1 \mathrm{U} / \mathrm{ml}$ thrombin in the presence of varying concentrations of isoquercetin ( 0 to $50 \mu \mathrm{M})$. Platelet releasates were immunoblotted for factor Va. As shown in Figure 6, we observed a dose-dependent reduction in factor Va following platelet activation. The reduction in factor Va generation paralleled the reduction in thrombin generation in experiments performed under the same conditions (Figure 6B). PDI activity in the presence of isoquercetin decreased over the same concentrations of isoquercetin (Figure 6B). At $10 \mu \mathrm{M}$ isoquercetin, no additional decrease in thrombin generation was observed when an inhibitory anti-factor Va antibody $(280 \mu \mathrm{g} / \mathrm{ml})$ was combined with isoquercetin $(43.7 \% \mathrm{vs} .50 .2 \%, P=0.66)$, suggesting that complete platelet factor Va inhibition is achieved at this isoquercetin concentration. Platelet $\alpha$-granule release of platelet factor 4 (PF4) did not demonstrate a similar effect, arguing against the observed reduction in factor Va representing a decrease in platelet activation and impaired secretion in response to 


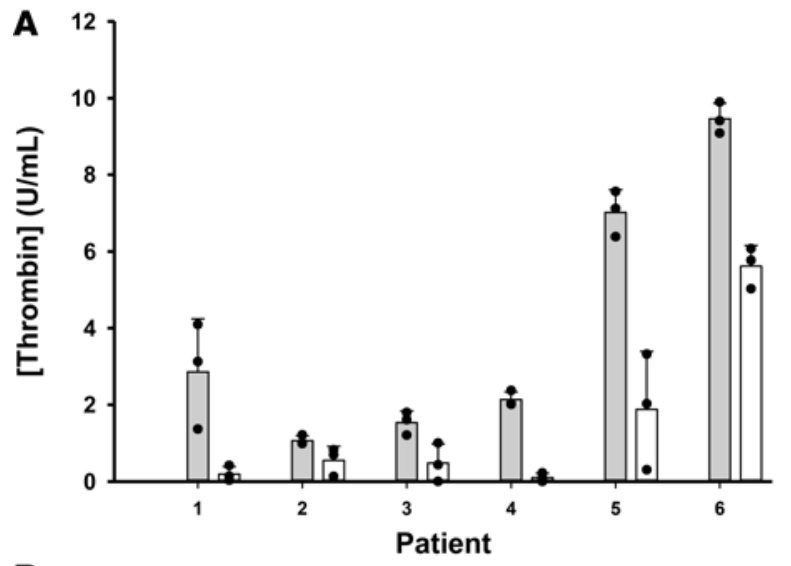

B

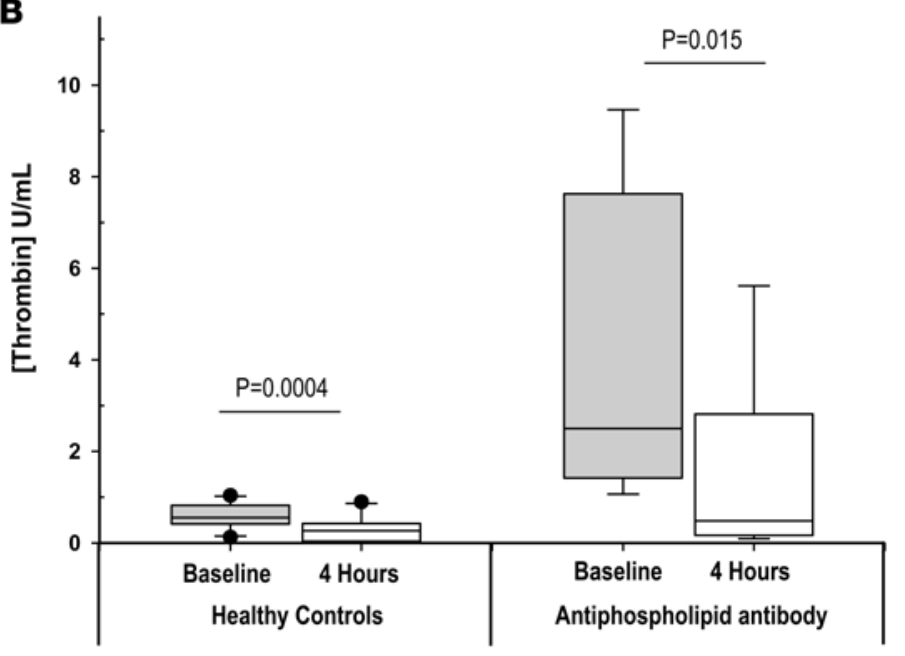

Figure 4. Inhibition of thrombin generation with isoquercetin in patients with elevated anti-phospholipid antibodies. (A) Six subjects with anti-phospholipid antibodies received an oral dose of 1,000 mg isoquercetin. Platelet-dependent thrombin generation was assayed before (gray bars) and 4 hours following (white bars) ingestion of isoquercetin $(1,000 \mathrm{mg})$. Bars represent the mean of triplicate values of individual subjects (dark circles) with error bars representing SD. (B) Box-andwhisker plot showing thrombin generation in healthy controls $(n=10)$ and anti-phospholipid antibody patients $(n=6)$ before (Baseline) and 4 hours after (4 hours) ingestion of 1,000 mg isoquercetin. Top and bottom of box represent the interquartile range along with median values, whiskers represent 95 th percentiles, and outliers are shown. $P$ values were determined using paired $t$ tests.

thrombin (Figure 6C). In the absence of platelets, isoquercetin (10 $\mu \mathrm{M})$ did not directly inhibit factor Xa or prothrombinase complex activity (Figure 6, D and E).

In order to determine whether the effect of isoquercetin on factor Va generation contributes to its inhibition of thrombin generation in human subjects, we analyzed patient plasma following the oral consumption of isoquercetin. Plasma samples collected from healthy participants before and 4 hours after ingestion of $1,000 \mathrm{mg}$ of isoquercetin ( $n=17$ ) were immunodepleted of factor V. Factor V-depleted plasma was reconstituted with healthy donor platelets and stimulated with low-dose thrombin. Platelet stimulation using factor V-depleted plasma yielded $45 \%$ less measured factor Va relative to non-factor $\mathrm{V}$-depleted plasma $(P<0.001)$. Thus, in these experiments approximately $55 \%$ of factor $\mathrm{Va}$ in plasma following platelet activation is derived from platelet stores. Following the oral administration of isoquercetin, we observed an overall $26 \%$ reduction in factor Va in non-factor Va-depleted plasma $(P<0.001)$ which corresponded with a $53 \%$ decrease in factor Va generated from platelets $(P<0.001$, Figure $7, \mathrm{~A}$ and B). These data confirm a significant effect of isoquercetin ingestion on the generation of factor Va following platelet activation. Considering that isoquercetin reduces platelet factor Va generation and similarly inhibits platelet-dependent thrombin generation in a PDI-dependent manner, we investigated whether the addition of factor Va in vitro restored platelet-dependent thrombin generation. The preincubation of $7 \mu \mathrm{g} / \mathrm{ml}$ factor Va prior to stimulation with low-dose thrombin significantly increased platelet-dependent thrombin generation $(P=0.04)$ to within $80 \%$ baseline of pretreatment levels (Figure $7 \mathrm{C}$ ). We conclude that isoquercetin-mediated inhibition of PDI activity significantly decreases platelet-dependent thrombin generation predominantly through a reduction in platelet-derived factor Va generation.

\section{Discussion}

Although PDI is primarily a resident protein of the ER and dense tubular system in endothelial cells and platelets, respectively, a small portion of PDI resides in granules and is released upon cell activation. This extracellular PDI serves an essential role in thrombus formation in vivo (2-4). The fortuitous observation that certain quercetin flavonoids inhibit PDI facilitated the investigation of extracellular PDI as a druggable antithrombotic target in humans (6). To the best of our knowledge, we report the first clinical study specifically designed to target extracellular PDI in humans.

Quercetin-3-rutinoside was the most potent small-molecule inhibitor of PDI identified in a high-throughput screen of biologically active compounds (6). Quercetin-3-rutinoside administered intravenously to mice abolished both platelet accumulation and fibrin generation at the site of a vascular injury, an effect that reversed completely following administration of recombinant PDI (6). Quercetin-3-rutinoside binds reversibly to the $b^{\prime}$ domain of PDI, leading to a conformational change that is detected by small-angle $\mathrm{x}$-ray scattering as a reduction in the molecular envelope (7). Structure-activity relationship studies identified several quercetin analogs with similar anti-PDI activity including isoquercetin. All quercetin flavonoid analogs assayed that contained a glycoside at the 3-C position of the quercetin backbone demonstrated anti-PDI activity (6). This 


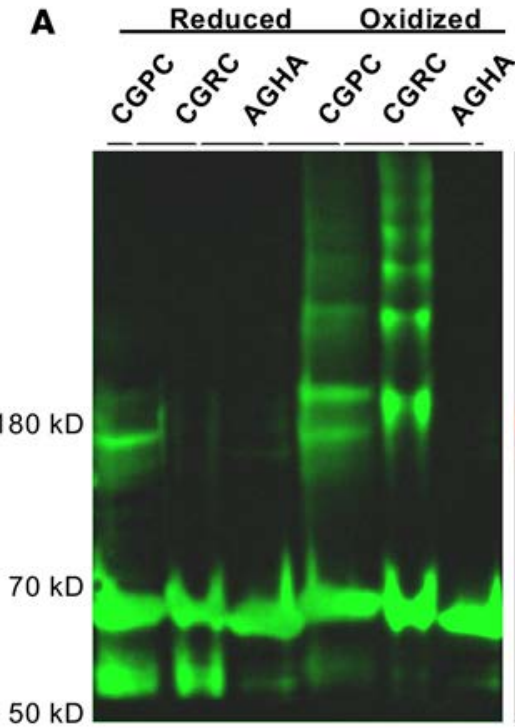

IB: FLAG

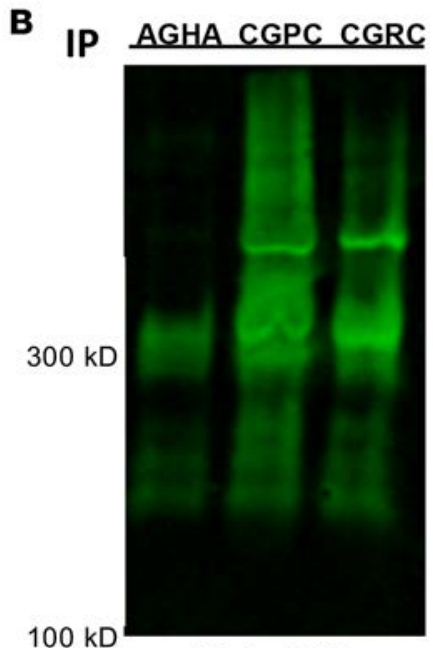

IB: Anti-FV
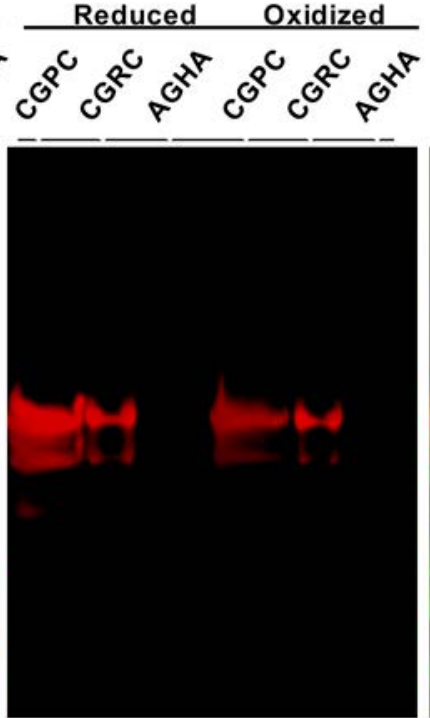

Factor V

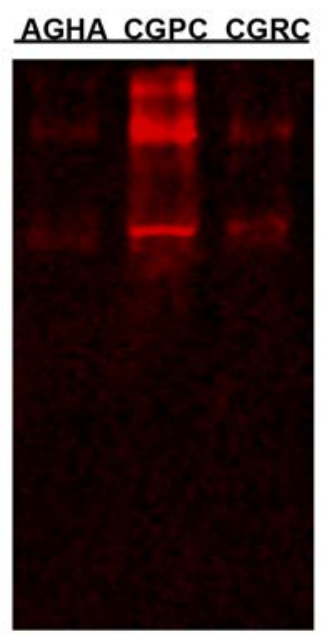

IB: Anti-MMN1
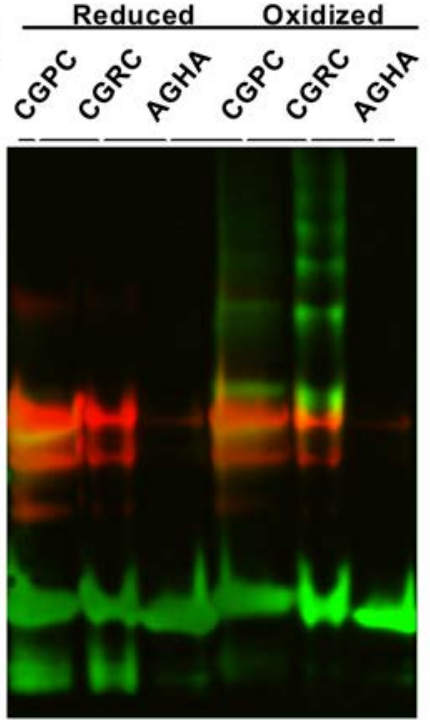

Merge

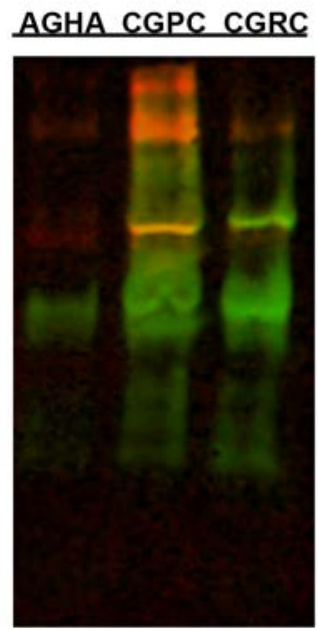

Merge

Figure 5. Factor $\mathbf{V}$ is a substrate of protein disulfide isomerase (PDI). (A) PDI trapping mutants react with factor $\mathrm{V}$ released from activated platelets. PDI variants (CGPC-PDI, CGRC-PDI, or AGHA-PDI) labeled with an N-terminal FLAC tag were either prereduced (Reduced) or preoxidized (Oxidized) prior to incubation with platelets. Washed platelets were stimulated with thrombin in the presence of 1 of the FLAG-tagged PDI variants. Platelets were separated from releasates by centrifugation and FLAG-tagged PDI constructs were pulled down using anti-FLAG antibody-coated beads. Precipitated proteins were resolved by polyacrylamide gel electrophoresis under nonreducing conditions, transferred to PVDF, and immunoblotted (IB) for both FLAC (green) and factor V (red). Overlapping bands are shown in yellow in the merge. (B) Evaluation of nonreduced samples indicates that factor $V(F V)$ trapped by PDI variants associates with multimerin-1 (MMN-1). Pulldowns of PDI variants (CGPC-PDI, CGRC-PDI, or ACHA-PDI) were performed as described in $\mathbf{A}$. Samples were resolved under nonreducing conditions using agarose electrophoresis to resolve high-molecular-weight complexes and transferred to PVDF membranes. Membranes were subsequently stained using anti-FV antibody (anti-FV) and antiMMN1 antibody (anti-MMN1). For all panels, representative images are shown for experiments performed in triplicate.

same glycoside that is required for the anti-PDI activity also impairs the ability of quercetin flavonoids to permeate cell membranes, thereby targeting extracellular PDI. Although the antithrombotic activity of quercetin3-rutinoside was initially tested in animal models, its absorption in humans is known to be poor $(8,30)$. We performed a comparative pharmacokinetic study between quercetin aglycone and isoquercetin and observed that oral administration of $1,000 \mathrm{mg}$ isoquercetin achieves plasma concentrations in line with the $\mathrm{IC}_{50}$ derived from PDI inhibitory assays in vitro (6). We confirmed on-target activity, as plasma obtained following administration of isoquercetin significantly reduced PDI activity in a time- and concentration-dependent manner. 
A

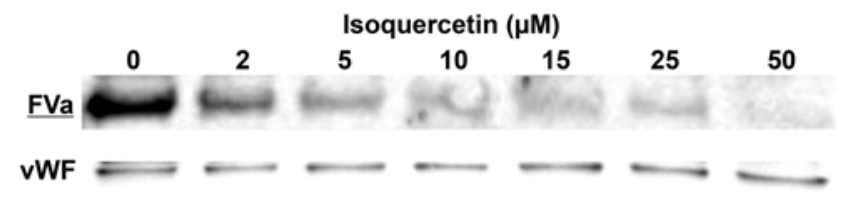

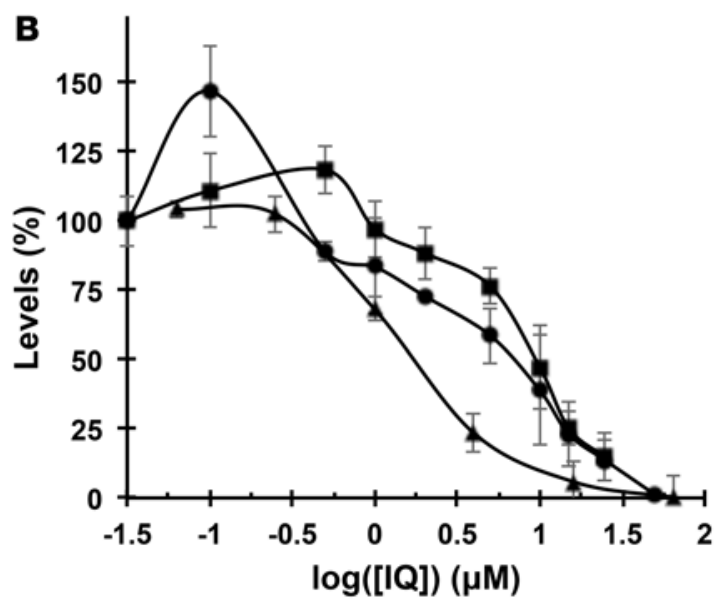

D

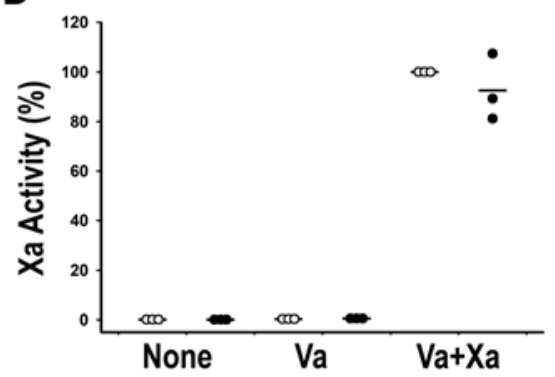

E

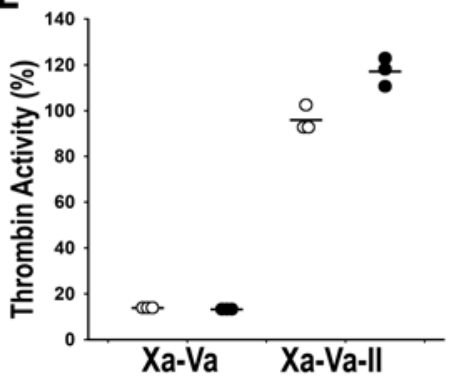

Figure 6. Isoquercetin-mediated protein disulfide isomerase inhibition targets platelet factor Va generation. (A) The generation of factor Va from platelets is decreased in the presence of isoquercetin in vitro. Washed platelets were stimulated with $0.1 \mathrm{U} / \mathrm{ml}$ thrombin in the presence of varying concentrations of isoquercetin. Proteins within releasates were stained with an antibody that specifically detects factor Va (FVa) and von Willebrand factor monomer was stained as a loading control (VWF). A representative image is shown for experiments performed in triplicate. (B) Comparison of thrombin generation, FVa generation, and PDI inhibition after washed platelets were stimulated using $0.1 \mathrm{U} / \mathrm{ml}$ thrombin in the presence of the indicated concentrations of isoquercetin (IQ). Thrombin generation (squares) was evaluated using a fluorescent substrate, FVa generation (circles) using a FVa-specific antibody, and PDI inhibition (triangles) by a di-eosin-GSSG assay. Symbols represent means of triplicates of activity ( \pm SD) compared with samples not exposed to isoquercetin. (C) The release of platelet factor 4 (PF4) from platelets is unaffected in the presence of isoquercetin. Washed platelets were incubated with $0.1 \mathrm{U} / \mathrm{ml}$ thrombin in the presence of the indicated concentrations of isoquercetin and platelet releasates isolated by centrifugation. Proteins within the releasates were resolved by gel electrophoresis and immunoblotted for PF4. Experiments were performed in triplicate and data represent band intensity compared with the no-isoquercetin control, mean \pm SD. (D) Isoquercetin does not directly inhibit factor Xa. Purified factor Xa (10 $\mu \mathrm{g} / \mathrm{ml})$ was combined with purified $\mathrm{FVa}(7 \mu \mathrm{g} / \mathrm{ml})$ and assayed for activity in the presence (black circles) or absence (white circles) of $10 \mu \mathrm{M}$ isoquercetin using a fluorescent cleavage substrate for Xa ( $\mathrm{Va}+\mathrm{Xa}$ ). Fluorescent substrate only (None) and FVa with substrate (Va) were included as controls. Triplicates (with lines representing means $\pm S D$ ) represent activity compared with samples not exposed to isoquercetin. (E) Prothrombin activation is not inhibited by isoquercetin. Thrombin activity was measured using a fluorescent cleavage substrate after combining factor Xa $(10 \mu \mathrm{g} / \mathrm{ml}), \mathrm{FVa}(7 \mu \mathrm{g} / \mathrm{ml})$, and prothrombin $(50 \mu \mathrm{g} / \mathrm{ml})(\mathrm{Xa}-\mathrm{Va}-\mathrm{II})$. Thrombin activity was measured after combining factors Xa and Va without prothrombin addition as a control (Xa-Va). Studies were performed in the presence (black circles) and absence (white circles) of isoquercetin. Experiments were performed in triplicate as shown (with means \pm SD) and data represent activity compared with samples not exposed to isoquercetin.

In order to evaluate the potential anticoagulant activity of PDI inhibition in humans, we measured thrombin generation in a coagulation assay dependent on PDI activity on the thrombin-platelet amplification loop (31). Following oral administration of isoquercetin, platelet-dependent thrombin generation in plasma decreased significantly in both the healthy cohort as well as in individuals with anti-phospholipid antibodies. The addition of recombinant PDI to the posttreatment plasma samples significantly increased thrombin generation, thus confirming the PDI-dependent nature of the observed isoquercetin-mediated decrease in thrombin generation. The marked inhibition in thrombin generation observed in patients with anti-phospholipid antibodies (and high baseline thrombin generation) is intriguing considering the heightened risk for arterial and venous thrombosis without a validated approach for primary prevention. We observed an approximately $90 \%$ normalization of thrombin generation with the addition of exogenous PDI to isoquercetin-treated plasma, thus indicating a small contribution for off-target anticoagulant activity of the drug. Our group is working to identify and characterize more specific small-molecule inhibitors of 
A
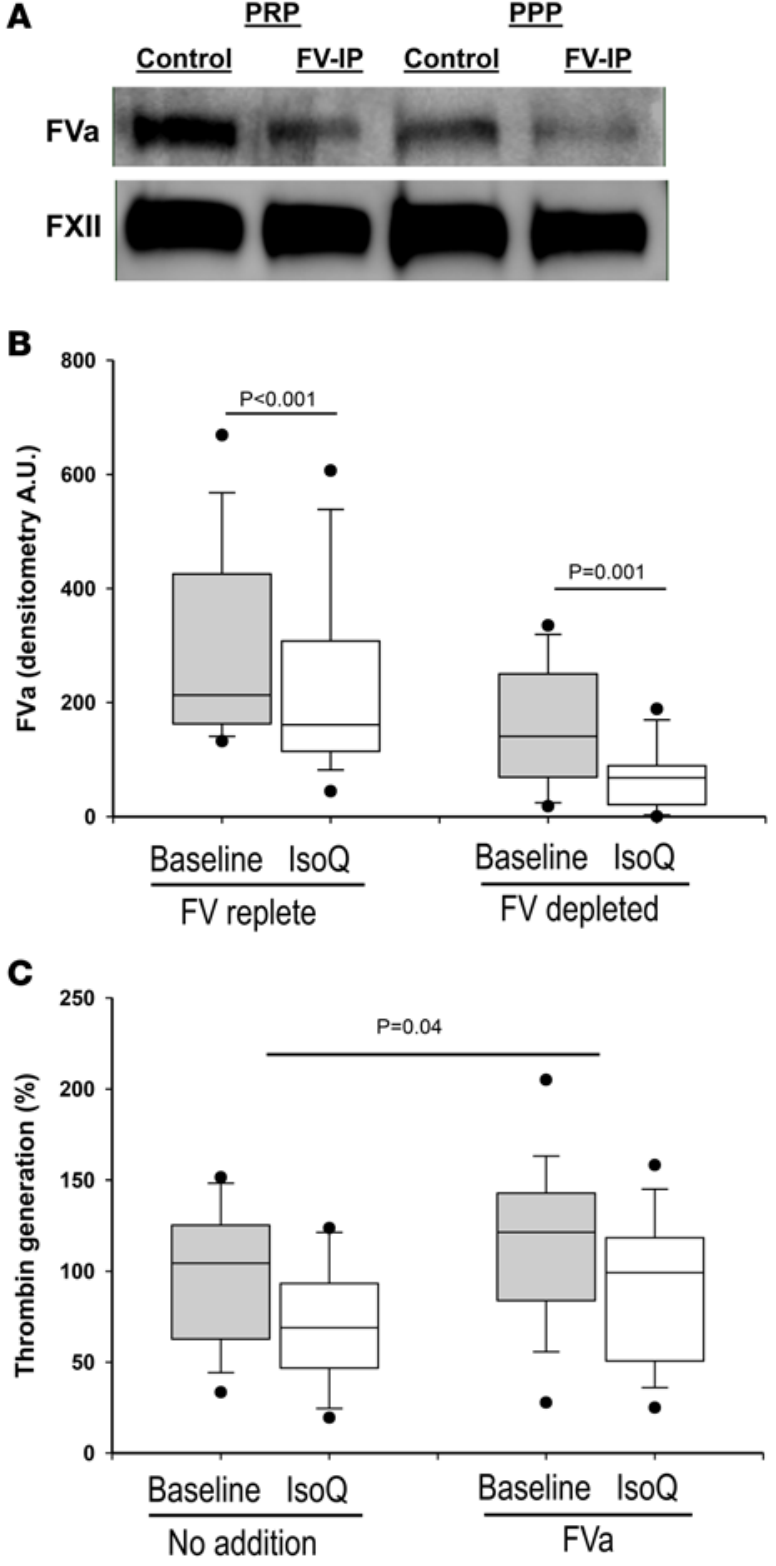

Figure 7. Factor $\mathbf{V}$ activation following ingestion of isoquercetin. (A) Plateletrich plasma (PRP) samples and platelet-poor plasma (PPP) samples were either untreated (Control) or depleted of plasma factor V (FV-IP) using an antibody directed against FV. Samples were subsequently stimulated with $0.1 \mathrm{U} / \mathrm{ml}$ thrombin and subjected to centrifugation. Proteins in the supernatant were resolved by gel electrophoresis, transferred to PVDF, and stained for factor Va (FVa). Staining of samples using anti-factor XII antibody was performed as a loading control (FXII). A representative blot is shown for experiments run for every patient $(n=$ 17). (B) The generation of FVa from platelets is decreased in the plasma of a subject following ingestion of isoquercetin. Plasma samples were obtained from 17 healthy participants before (baseline, gray boxes) and 4 hours after (IsoQ, white boxes) ingestion of $1,000 \mathrm{mg}$ isoquercetin. Samples were either left untreated (FV replete) or depleted of FV (FV depleted) and processed as described in $\mathbf{A}$. FVa staining in immunoblots was quantified by densitometry. Top and bottom of box-and-whisker plots represent the interquartile range along with median values, whiskers represent 95th percentiles, and outliers are shown. $P$ values represent paired $t$ test. (C) Isoquercetin-mediated reduction in platelet-dependent thrombin generation is reversed with FVa. Plasma samples were obtained from 17 healthy participants before (gray boxes) and 4 hours after (white boxes) ingestion of $1,000 \mathrm{mg}$ isoquercetin. Samples were then incubated with no addition (No addition) or $7 \mu \mathrm{g} / \mathrm{ml} \mathrm{FVa} \mathrm{(FVa)} \mathrm{and} \mathrm{evaluated} \mathrm{for} \mathrm{platelet-dependent} \mathrm{thrombin}$ generation. Top and bottom of box-and-whisker plots represent the interquartile range along with median values, whiskers represent 95 th percentiles, and outliers are shown. $P$ values represent 1-sample $t$ test of the Baseline/IsoQ ratios with and without FVa to test the null hypothesis that these ratios are the same.

PDI, which may enable a more targeted investigation of the thrombotic mechanisms under regulatory control of PDI in vivo $(32,33)$.

PDI activity has previously been shown to influence platelet-dependent thrombin generation, which we now show is dependent on PDI-mediated activation of platelet factor V. Although platelet-derived factor Va only accounts for $20 \%$ of the total amount of factor $\mathrm{V}$ measured in plasma, this pool plays a critical role in supporting hemostasis. Thrombin generation in the presence of normal platelets does not require plasma factor $\mathrm{V}$ and endogenous thrombin potential is not significantly reduced in patients with congenital factor $\mathrm{V}$ deficiency (absent plasma factor Va but measurable platelet factor Va) (34). In our experiments using human plasma depleted of factor $\mathrm{V}$, we observed an approximately $75 \%$ reduction in platelet-generated factor Va following isoquercetin ingestion. This reduction in platelet factor Va generation paralleled inhibition of PDI. The significant decrease in generation of factor Va following isoquercetin exposure was not due to decreased platelet activation, direct alteration of prothrombin or factor $\mathrm{Va}$, or the direct inhibition of thrombin or factor Xa.

The precise mechanism by which PDI influences the generation of platelet-dependent factor Va has yet to be elucidated. Factor V contains 18 cysteine residues: 14 form intramolecular disulfide bridges and 4 are unpaired, free sulfhydryl groups, 2 of which are readily accessible (35). Modification of free sulfhydryls within factor Va does not alter the rate of prothrombin activation (36). Factor V is stored within platelet $\alpha$-granules in complex with multimerin partially through disulfide linkages (37). Multimerin-1 in complex with factor $\mathrm{V}$ inhibits thrombin generation (38). Further studies, however, are necessary to determine whether PDI mediates the release of factor V/Va from multimerin following platelet activation. Although the generation of platelet-derived factor Va appears to be the dominant driver in the PDImediated reduction in platelet-dependent thrombin generation, evidence points against this being the only PDI substrate influencing thrombin generation in this assay. PDI-mediated activation of platelet integrins such as GPIb and $\alpha \operatorname{Ilb} \beta 3$ integrin or other coagulation targets (22) may explain the observation that restoration of thrombin generation by incubation with exogenous factor Va was incomplete, achieving $85 \%$ of baseline levels (Figure 7C). Furthermore, thrombus formation in vivo is considerably more complex than the platelet-dependent thrombin generation assay, which we use as a convenient functional measure of anti-PDI activity in human subjects. PDI substrates on endothelium and leukocytes could also contribute 
to thrombus formation in vivo. Surface receptors and adhesion proteins may serve more prominent roles under flow conditions. Nonetheless, the finding that PDI inhibition blocks platelet-dependent thrombin generation in human subjects provides what we believe to be the first mechanistic evidence of how PDI inhibition could impair thrombosis in human studies.

Despite an expanding list of antithrombotics available for therapeutic use, thrombotic disease remains the leading cause of mortality in developed countries. PDI inhibitors represent a novel multitarget anticoagulant that inhibits both platelet aggregation and fibrin generation with the potential to impact the management of complex thrombotic disorders such as myocardial infarction and cancer-associated thrombosis. There is considerable epidemiologic evidence linking quercetin-rich diets and improved cardiovascular outcomes. For instance, in a large prospective study of approximately 100,000 adults in the United States, the highest quintile of flavonoid consumption was associated with an approximately $20 \%$ decrease in cardiovascular deaths (adjusted risk ratio $0.82,95 \%$ CI $0.73-0.92, P=0.01$ ) (39). This report of pharmacodynamic inhibition of PDI with isoquercetin along with underlying mechanistic studies identifying platelet factor Va as a dominant anticoagulant substrate of PDI represents an important first step in the development of a novel class of antithrombotic agents targeting PDI. Based on these data we initiated advanced-stage clinical trials to assess the efficacy of isoquercetin to prevent thrombosis in high-risk cancer patients (NCT02195232).

\section{Methods}

Eligibility. Enrollment into the healthy cohort only included participants without known liver, kidney, cardiac, or other chronic illness. Participants were excluded if taking anticoagulants or antiplatelet agents (including nonsteroidal antiinflammatory medications). Study subjects were instructed to avoid quercetinrich foods for 72 hours prior to administration of study drug. A flavonoid-free lunch was provided at 4 hours after the initial blood draw. Study subjects were allowed to participate in more than 1 cohort following a minimum of a 2 -week washout period. For enrollment into the cohort with anti-phospholipid antibodies, study subjects were required to have at least 1 positive anti-phospholipid antibody assay (lupus anticoagulant, anticardiolipin antibody IgM or IgG > 40 GPL, or anti- $\beta 2$ glycoprotein- 1 antibody titer $>$ 35 units) confirmed at least 8 weeks apart.

Study procedures. Quercetin aglycone and isoquercetin were provided by Quercegen Pharma. The cohorts including healthy subjects were: (a) $500 \mathrm{mg}$ quercetin aglycone $(n=5)$; (b) $500 \mathrm{mg}$ quercetin aglycone with $500 \mathrm{mg}$ ascorbic acid and $20 \mathrm{mg}$ niacin ( $n=5$ ); (c) $500 \mathrm{mg}$ isoquercetin $(n=5)$; (d) $500 \mathrm{mg}$ isoquercetin with $500 \mathrm{mg}$ ascorbic acid and $20 \mathrm{mg}$ niacin $(n=5)$; and (e) 1,000 $\mathrm{mg}$ isoquercetin with $1,000 \mathrm{mg}$ ascorbic acid and $40 \mathrm{mg}$ niacin $(n=10)$. Study subjects with anti-phospholipid antibodies received 1,000 $\mathrm{mg}$ isoquercetin with $1,000 \mathrm{mg}$ ascorbic acid and $40 \mathrm{mg}$ niacin $(n=6)$. For the healthy cohorts $(\mathrm{a}-\mathrm{e})$, blood was drawn prior to the administration of study drug and at 8 time points over a 24 -hour period $(0,0.5,1$, $2,4,6,8$, and 24 hours). For patients with anti-phospholipid antibodies, blood was drawn at 0 and 4 hours only. Total plasma quercetin was measured by David Nieman at North Carolina Research Campus by previously described methods (40).

Di-eosin-GSSG Assay. Preparation of di-eosin-GSSG was performed as described (41). The platelet-poor plasma was filtered through a Millipore $30-\mathrm{kDa}$ cutoff filter at $14,000 \mathrm{~g}$ and the filtrate incubated with 10 $\mu \mathrm{M}$ GSSG for 20 minutes at room temperature. Human recombinant PDI dual tagged with FLAG and streptavidin-binding peptide was expressed and isolated from E. coli (BL21) by affinity chromatography with streptavidin-linked agarose resin. Purified recombinant PDI was added at varying concentrations $(0$, 50,100 , and $250 \mathrm{nM}$ ) to filtered GSSG-treated plasma with $5 \mu \mathrm{M}$ DTT and $150 \mathrm{nM}$ di-eosin-GSSG. Enzymatic activity was monitored through the fluorescence of eosin (EGSH), which was excited at $525 \mathrm{~nm}$ and emission was recorded at $545 \mathrm{~nm}$. Linear rates were obtained and converted to units of enzymatic activity per $\mathrm{ml}$ of PDI stock. Once standardized, $2 \mathrm{U} / \mathrm{ml}$ PDI was added to $5 \mu \mathrm{M}$ DTT and $150 \mathrm{nM}$ di-eosinGSSG in a total volume of $80 \mu 1$ with GSSG-treated filtered plasma. Percentage inhibition was assessed by the difference in measured units of activity from those of the standard. For the $\mathrm{IC}_{50}$ determination, 100 $\mu \mathrm{M}$ recombinant PDI was incubated in GSSG-treated filtered plasma for 5 minutes with concentrations of quercetin analog ranging from $20 \mathrm{nM}$ to $3 \mathrm{mM}$ in GSSG-treated filtered plasma and then assayed for enzymatic activity as described. Percentage inhibition was normalized to the no-addition control (100\%).

Thrombin generation. Whole blood was drawn from volunteers just before ( 0 hours) and 4 hours after oral ingestion of $1,000 \mathrm{mg}$ isoquercetin. Whole blood was spun at $300 \mathrm{~g}$ for 20 minutes to isolate platelet-rich 
plasma (PRP). Platelet-free plasma (PFP) was generated by centrifuging PRP twice at 2,800 $g$ for 10 minutes. $\mathrm{PRP}$ was diluted to a platelet concentration of 250,000 platelets per microliter with platelet-poor plasma in the presence of $5 \mathrm{mM}$ Gly-Pro-Arg-Pro peptide (Thermo Fisher) and $6 \mathrm{mM} \mathrm{CaCl}_{2}$, and then stimulated with 0.1 $\mathrm{U} / \mathrm{ml}$ thrombin. For the thrombin generation rescue experiments, $50 \mu \mathrm{M}$ PDI was also added. PRP samples were incubated for 90 minutes at room temperature with rotation, and thrombin levels were measured using cleavage of a fluorescent thrombin-specific substrate (Haemtech SN-20 Boc-L-FPR-ANSNH-C2H5) by excitation at $352 \mathrm{~nm}$ and emission as a function of time at $470 \mathrm{~nm}$. PRP was diluted 1:4 into $1 \times$ PBS containing $100 \mu \mathrm{M}$ fluorescent substrate and $12.5 \mathrm{mM}$ EDTA. Rates of substrate cleavage were used to calculate the concentration of thrombin in each sample at time zero and 4 hours after isoquercetin ingestion.

PDI variant generation and platelet factor $V$ trapping. The PDI gene (PDIA1) was inserted into the pT7FLAG-SBP-1 vector purchased from Sigma-Aldrich. Point mutations in the PDI sequence were made in subsequent rounds using the Site-Directed Mutagenesis Quick Change Kit available from New England Biolabs as previously described (22). PDI protein was expressed in the BL21 strain of E. coli, first grown in $\mathrm{LB}$ media at $37^{\circ} \mathrm{C}$ until induced at $25^{\circ} \mathrm{C}$ with $1 \mathrm{mM}$ isopropyl $\beta$-D-1-thiogalactopyranoside. Cells were lysed using the BPER Bacterial Protein Extraction Kit from Thermo Fisher. Clarified lysates were incubated with high-capacity streptavidin-coated agarose beads overnight at $4^{\circ} \mathrm{C}$, washed with at least 10 column volumes of $1 \times$ Tris-buffered saline with $0.1 \%$ Tween-20 (TBST), and eluted with $2 \mathrm{mM}$ biotin in TBST. Eluates were dialyzed 3 times against $1 \times$ PBS, concentrated, and stored frozen at $-80^{\circ} \mathrm{C}$.

Purified PDI variants or inactive PDI were added to washed platelets $\left(1 \times 10^{9}\right.$ to $\left.3 \times 10^{9}\right)$ in the presence or absence of $1 \mathrm{mM} \mathrm{CaCl}_{2}, 10 \mathrm{mM} \mathrm{MnCl}_{2}$, and $1.0 \mathrm{U} / \mathrm{ml}$ thrombin and allowed to incubate at room temperature for 3 minutes. Hirudin $(20 \mathrm{U} / \mathrm{ml})$ was added and incubated for 1 minute. NEM $(20 \mathrm{mM})$ was added and allowed to incubate at room temperature protected from light for 30 minutes. The platelet suspension was then spun down at $1,000 \mathrm{~g}$ for 10 minutes and separated into releasate and pellet fractions. The releasate fraction was spun for an additional 5 minutes at 2,800 $\mathrm{g}$. The pellet fraction was resuspended in $1 \mathrm{ml}$ of cold $20 \mathrm{mM}$ Tris (pH 7.4), 2\% Triton X-100, $20 \mathrm{mM}$ EGTA, $20 \mathrm{mM} \mathrm{MgCl}$, 2 mM ATP, $20 \mathrm{mM}$ NEM, $0.025 \mathrm{mg} / \mathrm{ml}$ cytochalasin D, $0.8 \mathrm{mM}$ PMSF, and 1× Protein Inhibitor Cocktail (Thermo Fisher), and incubated on ice for 30 minutes with regular inversion. The platelet lysate was then mixed with $1 \mathrm{ml}$ cold $2 \mathrm{M}$ Tris ( $\mathrm{pH}$ 7.0), $0.5 \mathrm{mM}$ ATP, $0.2 \mathrm{mM} \mathrm{MgCl}_{2}$, and $20 \mathrm{mM} \mathrm{NEM}$, and incubated on ice for $30 \mathrm{~min}-$ utes with regular inversion. The lysate was then centrifuged at $13,100 \mathrm{~g}$ for 10 minutes at $4^{\circ} \mathrm{C}$. PDI-substrate complexes were captured from clarified lysates or releasates using anti-FLAG magnetic beads (M2 monoclonal, Sigma-Aldrich) by inversion overnight at $4^{\circ} \mathrm{C}$ in the dark. The magnetic beads were separated and rinsed 3 times with $20 \mathrm{mM}$ HEPES (pH 7.4), 5\% Triton X-100, and $500 \mathrm{mM} \mathrm{NaCl}$. Complexes were eluted by heating at $95^{\circ} \mathrm{C}$ for 15 minutes in Laemmli buffer.

Antibodies. All primary antibodies were directed toward human antigens except for FLAG antibodies. All antibodies were used at manufacturer-recommended dilutions unless otherwise stated. For immunoprecipitation of FLAG-tagged species, mouse anti-FLAG M2 monoclonal (Sigma-Aldrich, M8823) was used. For identification of FLAG-tagged species during trapping experiments, rabbit anti-FLAG (Abcam, ab1162) was used. For identification of factor V during trapping experiments, sheep anti-factor V polyclonal (Thermo Fisher, PA1-43041) was used. For identification of multimerin-1 (MMN1) during trapping experiments, mouse anti-MMN1 monoclonal, JS1, a gift from Catherine Hayward (McMaster University, Ontario, Canada) was used. For immunoprecipitation of factor V from plasma, we used mouse anti-factor V monoclonal (Haematologic Technologies, AHV-5146). For identification of factor Va via Western blot, mouse anti-factor Va monoclonal (Haematologic Technologies, AHV-5112) was used. For inhibition of factor $\mathrm{V}$ during thrombin generation, we used a mouse anti-factor $\mathrm{V}$ monoclonal (Haematologic Technologies, AHV-5101).

Clotting assays. All PT and TT assays were performed on a Stago Start 4 instrument using the manufacturer's standard protocol and STA Neoplastin CI Plus (Stago).

Measuring factor Xa activity. Purified factor Xa (Haemtech) was diluted to $10 \mu \mathrm{g} / \mathrm{ml}$ in PBS along with 7 $\mu \mathrm{g} / \mathrm{ml}$ factor Va in the presence or absence of isoquercetin $(10 \mu \mathrm{M})$ and assayed for activity against either a synthetic factor Xa cleavage substrate (Haemtech, SN-7), or purified prothrombin (Haemtech). For assaying activity against prothrombin, $50 \mu \mathrm{g} / \mathrm{ml}$ prothrombin was incubated with factor $\mathrm{Xa}$, and then the solution was assayed for thrombin activity using a synthetic thrombin cleavage substrate (Haemtech, SN-20). Cleavage of both substrates was monitored over time via fluorescence, exciting at $352 \mathrm{~nm}$ and measuring emission over time at $470 \mathrm{~nm}$ in a Synergy 4 Fluorescent Plate Reader (BioTek). 
Statistics. Pharmacokinetic analysis was performed using STATA software. Statistical comparisons for intra-dosing cohorts (e.g., quercetin with or without ascorbic acid) were performed using a paired nonparametric analysis (Wilcoxon signed-rank test) for the AUC and $\mathrm{C}_{\max }$. Comparisons between baseline and subsequent measurement of PDI activity and thrombin generation were performed using 2-tailed, paired $t$-test analysis. Statistical significance was defined as a $P$ value less than 0.05 . ANOVA with Sheffe's correction for multiplicity testing was performed for PDI and factor $V$ rescue experiments.

Study approval. The protocol was approved by the IRB at Beth Israel Deaconess Medical Center. All study subjects provided written informed consent prior to the initiation of study procedures.

\section{Author contributions}

JDS designed and conducted experiments, acquired data, analyzed data, and contributed to writing of the manuscript. DN and MP performed statistical analyses. BF conceived of substrate trapping experiments, contributed to writing of the manuscript, and edited the manuscript. RF and JIZ conceived of pharmacokinetic and pharmacodynamics studies, wrote the manuscript, and edited the manuscript. JIZ designed clinical studies.

\section{Acknowledgments}

This work was supported by a grant from the National Heart, Lung, and Blood Institute (U54 HL112302, HL007917, and HL125275) as well as research support from Quercegen Pharma. Research in patients with anti-phospholipid antibodies was supported, in part, by the Lupus Research Institute. Clinical research support was provided in part by the Harvard Catalyst, the Harvard Clinical and Translational Science Center (National Center for Research Resources and the National Center for Advancing Translational Sciences, NIH award 8UL1TR000170-05 and financial contributions from Harvard University and its affiliated academic health care centers). Anti-multimerin-1 antibody was a gift from the Hayward group (McMaster University, Ontario, Canada). The content is solely the responsibility of the authors and does not necessarily represent the official views of Harvard Catalyst, Harvard University and its affiliated academic health care centers, or the NIH.

Address correspondence to: Jeffrey I. Zwicker or Robert Flaumenhaft, Division of Hemostasis and Thrombosis, Beth Israel Deaconess Medical Center, Harvard Medical School, 330 Brookline Avenue, Boston, Massachusetts 02215, USA. Phone: 617.667.9299; E-mail: jzwicker@bidmc.harvard.edu (J.I. Zwicker). Phone: 617.735.4001; E-mail: rflaumen@bidmc.harvard.edu (R. Flaumenhaft).

1. Furie B, Flaumenhaft R. Thiol isomerases in thrombus formation. Circ Res. 2014;114(7):1162-1173

2. Cho J, Furie BC, Coughlin SR, Furie B. A critical role for extracellular protein disulfide isomerase during thrombus formation in mice. J Clin Invest. 2008;118(3):1123-1131.

3. Reinhardt $\mathrm{C}$, et al. Protein disulfide isomerase acts as an injury response signal that enhances fibrin generation via tissue factor activation. J Clin Invest. 2008;118(3):1110-1122.

4. Zhou J, et al. The C-terminal CGHC motif of protein disulfide isomerase supports thrombosis. J Clin Invest. 2015;125(12):4391-4406.

5. Kim K, et al. Platelet protein disulfide isomerase is required for thrombus formation but not for hemostasis in mice. Blood. 2013;122(6):1052-1061.

6. Jasuja R, et al. Protein disulfide isomerase inhibitors constitute a new class of antithrombotic agents. J Clin Invest. 2012;122(6):2104-2113.

7. Lin L, et al. Quercetin-3-rutinoside inhibits protein disulfide isomerase by binding to its b'x domain. J Biol Chem. 2015;290(39):23543-23552.

8. Hollman PC, de Vries JH, van Leeuwen SD, Mengelers MJ, Katan MB. Absorption of dietary quercetin glycosides and quercetin in healthy ileostomy volunteers. Am J Clin Nutr. 1995;62(6):1276-1282.

9. Hollman PC, et al. Relative bioavailability of the antioxidant flavonoid quercetin from various foods in man. FEBS Lett. 1997;418(1-2):152-156.

10. Erlund I, et al. Pharmacokinetics of quercetin from quercetin aglycone and rutin in healthy volunteers. Eur J Clin Pharmacol. 2000;56(8):545-553.

11. Manach C, Williamson G, Morand C, Scalbert A, Rémésy C. Bioavailability and bioefficacy of polyphenols in humans. I. Review of 97 bioavailability studies. Am J Clin Nutr. 2005;81(1 Suppl):230S-242S.

12. Hertog MG, Feskens EJ, Hollman PC, Katan MB, Kromhout D. Dietary antioxidant flavonoids and risk of coronary heart disease: the Zutphen Elderly Study. Lancet. 1993;342(8878):1007-1011.

13. Keli SO, Hertog MG, Feskens EJ, Kromhout D. Dietary flavonoids, antioxidant vitamins, and incidence of stroke: the Zutphen study. Arch Intern Med. 1996;156(6):637-642.

14. Burgess JK, et al. Physical proximity and functional association of glycoprotein 1balpha and protein-disulfide isomerase on the 
platelet plasma membrane. J Biol Chem. 2000;275(13):9758-9766.

15. Lahav J, et al. Enzymatically catalyzed disulfide exchange is required for platelet adhesion to collagen via integrin alpha2beta1. Blood. 2003;102(6):2085-2092

16. Essex DW, Li M, Miller A, Feinman RD. Protein disulfide isomerase and sulfhydryl-dependent pathways in platelet activation. Biochemistry. 2001;40(20):6070-6075.

17. Giannakopoulos B, et al. Factor XI is a substrate for oxidoreductases: enhanced activation of reduced FXI and its role in antiphospholipid syndrome thrombosis. J Autoimmun. 2012;39(3):121-129.

18. Chen VM, Ahamed J, Versteeg HH, Berndt MC, Ruf W, Hogg PJ. Evidence for activation of tissue factor by an allosteric disulfide bond. Biochemistry. 2006;45(39):12020-12028.

19. Ahamed J, et al. Disulfide isomerization switches tissue factor from coagulation to cell signaling. Proc Natl Acad Sci USA. 2006;103(38):13932-13937.

20. Kothari H, Nayak RC, Rao LV, Pendurthi UR. Cystine 186-cystine 209 disulfide bond is not essential for the procoagulant activity of tissue factor or for its de-encryption. Blood. 2010;115(21):4273-4283.

21. Versteeg HH, Ruf W. Tissue factor coagulant function is enhanced by protein-disulfide isomerase independent of oxidoreductase activity. J Biol Chem. 2007;282(35):25416-25424.

22. Stopa JD, Furie BC, Furie B. Identifying substrates of protein disulfide isomerase using enzyme variants with altered redox potential [ISTH abstract PO551-WED]. J Thromb Haemost. 2015;13(suppl 2):1-977.

23. Dargaud Y, Luddington R, Baglin T. Platelet-dependent thrombography: a method for diagnostic laboratories. Br J Haematol. 2006;134(3):323-325.

24. Panova-Noeva M, et al. Platelet-induced thrombin generation by the calibrated automated thrombogram assay is increased in patients with essential thrombocythemia and polycythemia vera. Am J Hematol. 2011;86(4):337-342.

25. Tournier A, et al. Calibrated automated thrombography demonstrates hypercoagulability in patients with idiopathic pulmonary arterial hypertension. Thromb Res. 2010;126(6):e418-e422.

26. Lisman T, Moschatsis S, Adelmeijer J, Nieuwenhuis HK, De Groot PG. Recombinant factor VIIa enhances deposition of platelets with congenital or acquired alpha IIb beta 3 deficiency to endothelial cell matrix and collagen under conditions of flow via tissue factor-independent thrombin generation. Blood. 2003;101(5):1864-1870.

27. Wielders SJ, Broers J, ten Cate H, Collins PW, Bevers EM, Lindhout T. Absence of platelet-dependent fibrin formation in a patient with Scott syndrome. Thromb Haemost. 2009;102(1):76-82.

28. Balmer Y, Koller A, del Val G, Manieri W, Schürmann P, Buchanan BB. Proteomics gives insight into the regulatory function of chloroplast thioredoxins. Proc Natl Acad Sci USA. 2003;100(1):370-375.

29. Darby NJ, Creighton TE. Characterization of the active site cysteine residues of the thioredoxin-like domains of protein disulfide isomerase. Biochemistry. 1995;34(51):16770-16780.

30. Kienzler JL, Sallin D, Schifflers MH, Ghika A. Pharmacokinetics of mono-3'- and mono-4'-0-(beta-hydroxyethyl)-rutoside derivatives, after single doses of Venoruton powder in healthy volunteers. Eur J Clin Pharmacol. 2002;58(6):395-402.

31. Jurk K, Lahav J, VAN Aken H, Brodde MF, Nofer JR, Kehrel BE. Extracellular protein disulfide isomerase regulates feedback activation of platelet thrombin generation via modulation of coagulation factor binding. J Thromb Haemost. 2011;9(11):2278-2290.

32. Flaumenhaft R, Furie B, Zwicker JI. Therapeutic implications of protein disulfide isomerase inhibition in thrombotic disease. Arterioscler Thromb Vasc Biol. 2015;35(1):16-23.

33. Bekendam RH, et al. A substrate-driven allosteric switch that enhances PDI catalytic activity. Nat Commun. 2016;7:12579.

34. Duckers C, et al. Residual platelet factor V ensures thrombin generation in patients with severe congenital factor V deficiency and mild bleeding symptoms. Blood. 2010;115(4):879-886.

35. Xue J, Kalafatis M, Silveira JR, Kung C, Mann KG. Determination of the disulfide bridges in factor Va heavy chain. Biochemistry. 1994;33(44):13109-13116.

36. Krishnaswamy S, Mann KG. The binding of factor Va to phospholipid vesicles. J Biol Chem. 1988;263(12):5714-5723.

37. Hayward CP, et al. Factor V is complexed with multimerin in resting platelet lysates and colocalizes with multimerin in platelet alpha-granules. J Biol Chem. 1995;270(33):19217-19224

38. Jeimy SB, et al. Multimerin 1 binds factor V and activated factor V with high affinity and inhibits thrombin generation. Thromb Haemost. 2008;100(6):1058-1067.

39. McCullough ML, Peterson JJ, Patel R, Jacques PF, Shah R, Dwyer JT. Flavonoid intake and cardiovascular disease mortality in a prospective cohort of US adults. Am J Clin Nutr. 2012;95(2):454-464.

40. Jin F, Nieman DC, Shanely RA, Knab AM, Austin MD, Sha W. The variable plasma quercetin response to 12-week quercetin supplementation in humans. Eur J Clin Nutr. 2010;64(7):692-697.

41. Raturi A, Vacratsis PO, Seslija D, Lee L, Mutus B. A direct, continuous, sensitive assay for protein disulphide-isomerase based on fluorescence self-quenching. Biochem J. 2005;391(Pt 2):351-357. 